\title{
Identificação e classificação de riscos na implantação da produção enxuta
}

\author{
Giuliano Almeida Marodin ${ }^{\mathrm{a}}$, Tarcísio Abreu Saurin ${ }^{\mathrm{b}}$, Carla Schwengber ten Caten ${ }^{\mathrm{b}}$ \\ aniversity of South Carolina, Columbia, SC, Estados Unidos \\ bUniversidade Federal do Rio Grande do Sul, Porto Alegre, RS, Brasil \\ *gmarodin@moore.sc.edu
}

\begin{abstract}
Resumo
0 processo de implantação de sistemas de produção enxuta é complexo, demorado e sujeito a uma série de riscos. Este artigo tem como objetivo identificar e classificar os riscos presentes na implantação da produção enxuta (IPE). As quatro etapas da pesquisa foram: (i) revisão de literatura para identificação de riscos à IPE; (ii) desenvolvimento e aplicação de um questionário a 57 respondentes, que avaliou as percepções acerca da probabilidade de ocorrência e impacto de cada risco apontado pela literatura; (iii) análise dos resultados do questionário, que permitiu a classificação dos riscos por meio de uma análise fatorial exploratória; (iv) estudo de caso para aprofundar a compreensão das relações entre os riscos em uma empresa na qual trabalhavam três respondentes da survey. Quatorze riscos à IPE foram identificados e classificados em três grupos: (i) riscos associados ao papel das áreas de apoio; (ii) riscos associados ao papel da alta e média gerência; e (iii) riscos associados ao papel dos níveis operacionais. Uma vez que os riscos dentro de cada grupo são fortemente inter-relacionados, essa classificação pode contribuir para o planejamento e implantação de ações de controle destes.
\end{abstract}

Palavras-chave

Produção Enxuta. Gestão de riscos. Análise fatorial exploratória.

\section{Introdução}

Há várias décadas, iniciativas de implantação da produção enxuta (IPE), ou sistema lean, vêm sendo realizadas por empresas de diversos setores ao redor do mundo (Krafcit, 1988; Womack \& Jones, 1996; Hines et al., 2004). Uma pesquisa de âmbito mundial, conduzida pelo Lean Enterprise Institute (2007) com 2440 empresas pertencentes a uma base de dados desta organização, demonstrou que 59\% delas estavam em fase de planejamento ou iniciando a IPE em áreas-piloto. Tal difusão se deve, em parte, aos resultados positivos que o uso de práticas e princípios enxutos tem produzido (Taj \& Morosan, 2011), ainda que frequentemente as empresas tenham dificuldades em sustentar os ganhos em longo prazo (Bateman \& David, 2002). De fato, estudos realizados em diferentes países têm indicado que poucas empresas alcançam a maturidade ou resultados sustentáveis com a IPE (Baker, 2002; Blanchard, 2007; Bhasin, 2012; Marodin
\& Saurin, 2013). Essa situação decorre, em parte, da própria natureza da IPE, que é complexa, demorada e requer a alocação de uma quantidade substancial de recursos financeiros e humanos (Papadopoulou \& Özbayrak, 2005; Emiliani \& Stec, 2005; Lian \& Van Landeghem, 2007).

0 grande número de empresas em fases iniciais de implantação e um substancial nível de insucesso na IPE indicam a necessidade de aprofundar o conhecimento sobre os fatores que influenciam na implantação desse sistema (Bayo-Moriones et al., 2008). Tais fatores têm sido discutidos em pesquisas com diferentes orientações metodológicas, como estudos de caso (Lima \& Zawislak, 2003; Achanga et al., 2006; Scherrer-Rathje et al., 2009; Piercy \& Rich, 2009) e surveys (Cua et al., 2001; White \& Prybutok, 2001; Shah \& Ward, 2003; Bayo-Moriones et al., 2008; Sim \& Rogers, 2008; Freitas et al., 2014). 
Neste estudo, os fatores de impacto na IPE são reinterpretados e investigados sob a perspectiva da gestão de riscos, uma vez que isso induz à gestão sistemática destes sob a lógica PDCA (Planejar-Exec utar-Checar-Agir), além de induzir à investigação do contexto em que ocorre a IPE (Marodin et al., 2014). De acordo com PMl (Project Management Institute, 2004), risco é qualquer evento ou condição de incerteza que pode influenciar negativa ou positivamente nos objetivos de um projeto ou processo. A gestão de riscos visa entender e controlar os riscos que podem afetar um projeto, de modo a aumentar as chances de resultados positivos (Ritchie \& Brindley, 2007). Cabe enfatizar que o uso da perspectiva da gestão de riscos no estudo da IPE não significa que ela deve se entendida como um projeto. Um dos princípios-chave do lean é a melhoria contínua, o que implica a ausência de um fim no processo de implantação, como geralmente ocorre em projetos (Hines et al., 2004; Papadopoulou \& Özbayrak, 2005; Pettersen, 2009).

A ausência de pesquisas que usem a perspectiva da gestão de riscos na IPE é paradoxal, seja em trabalhos acadêmicos ou em experiências práticas das empresas. Uma vez que o lean pressupõe a solução de problemas através da lógica PDCA, a falta de um método para gerenciar os riscos na IPE indica que essa lógica não tem sido usada na própria implantação. Todo projeto ou processo apresenta riscos (Raz et al., 2002), os quais geralmente são gerenciados por intuição, experiência ou de forma reativa (Williams et al., 2006). A falta de estudos sobre a gestão de riscos na IPE leva a crer que estes são normalmente gerenciados dessa forma ou são ignorados.

Neste estudo são investigadas duas atividades da gestão de riscos na IPE: identificação e classificação. Diferentemente de trabalhos anteriores acerca de dificuldades para a IPE (Shah \& Ward, 2003; Papadopoulou \& Özbayrak, 2005; Emiliani \& Stec, 2005; Sim \& Rogers, 2008), no presente estudo essas etapas são investigadas em conjunto, sob a perspectiva da gestão de riscos. Em particular, a questão de pesquisa investigada neste trabalho pode ser enunciada como segue: quais são e como podem ser classificados os riscos ao processo de IPE? Essa questão é investigada com base em uma surveyjunto a funcionários que representam empresas do Sul do Brasil e um estudo de caso em uma empresa na qual trabalhavam três respondentes da survey.

\section{Gestão de riscos e classificação de riscos}

Embora qualquer projeto apresente riscos, cada projeto envolve diferentes tipos de riscos, com diferentes probabilidades de ocorrência e impactos (Raz et al., 2002). Segundo esses autores, uma vez que os riscos não podem ser completamente eliminados, os projetos devem possuir mecanismos de gestão destes. A gestão de riscos possui três objetivos (Ritchie \& Brindley, 2007): (a) minimizar a probabilidade de ocorrência de um evento negativo; (b) minimizar as consequências do evento, uma vez que ele tenha ocorrido; e (c) compreender os fatores que levaram a tal evento.

Existe certo consenso em relação aos princípios centrais da gestão de riscos, apesar de diferenças nos termos usados para designar as etapas desta (Norrman \& Jansson, 2004). De acordo com Williams et al. (2006), a gestão de riscos possui três etapas: (a) identificação de riscos; (b) análise de riscos, que envolve a compreensão destes; e (c) resposta aos riscos e monitoramento deles. Já Hallikas et al. (2004) dividem a etapa (c) em duas: uma de desenvolvimento e implantação de respostas aos riscos e outra com ênfase no monitoramento.

A literatura apresenta diferentes classificações de riscos, geralmente categorizando-os de acordo com a origem destes (Aloini et al., 2012). lsto ocorre pelo fato de que as medidas de resposta aos riscos são mais eficazes quando aplicadas a uma categoria de riscos, já que teria efeito em todos os riscos de mesma origem (Bannerman, 2008).

Tang (2006), por exemplo, classifica os riscos em: operacionais, quando eles decorrem de incertezas da operação em si; e drásticos, quando decorrem de fatores externos de grande impacto, tais como catástrofes naturais, falências de sistemas financeiros e ataques terroristas. Tal classificação é similar à de Ritchie \& Brindley (2000), que classificam os riscos como endógenos, originados na própria empresa, e exógenos, originados na cadeia de suprimentos. Sumner (2000) encontrou seis categorias para riscos ligados ao desenvolvimento de softwares, sendo elas: adaptação organizacional, combinação de habilidades, estrutura e estratégia gerencial, desenho do sistema de software, envolvimento e treinamento do usuário e planejamento e integração de tecnologia. No mesmo tema, Wallace et al. (2004) definem outros grupos, denominados de riscos de: equipe, ambiente organizacional, requisitos do sistema, planejamento e controle, usuário e complexidade. As classificações de riscos segundo sua prioridade também são comuns. Por exemplo, Hallikas et al. (2004) usam uma matriz de probabilidade e impacto para a classificação dos riscos em alta, média e baixa importância.

\section{Método de pesquisa}

\subsection{Delineamento da pesquisa}

A pesquisa foi realizada em quatro etapas: (a) revisão de literatura para identificação de riscos à IPE; (b) desenvolvimento e aplicação de um questionário para coletar dados sobre as percepções de funcionários 
de empresas da região Sul do Brasil sobre a severidade e probabilidade dos riscos identificados na etapa anterior; (c) análise dos resultados do questionário, enfatizando a classificação dos riscos, com base em uma Análise Fatorial Exploratória (AFE); (d) estudo de caso em uma das empresas representadas pelos respondentes da survey, permitindo ampliar a compreensão acerca das relações entre os riscos nos grupos.

A revisão de literatura, etapa (a), contribuiu para responder à primeira parte da questão de pesquisa, ou seja, quais são os riscos à IPE. A coleta de dados nas etapas (b) e (c) foi realizada por meio de uma survey, cujos resultados foram analisados por meio de uma AFE, possibilitando identificar grupos formados por riscos similares. As pesquisas baseadas em surveys são frequentemente usadas nos estágios iniciais de conhecimento de um fenômeno, quando são denominadas de surveys exploratórias (Malhotra \& Grover, 1998). Os resultados de tais surveys costumam ser úteis para refinar e identificar novas questões de pesquisa. No presente estudo, os resultados da survey indicaram que os riscos a IPE podem ser agrupados em três grupos, cada um com particularidades em termos das origens dos riscos e possíveis medidas de controle.

Entretanto, o uso da survey não foi suficiente para identificar por que e como os riscos dentro de cada grupo estavam relacionados. De fato, as surveys costumam contribuir pouco para responder questões dos tipos por que e como (Yin, 2003). A revisão de literatura, no caso deste estudo, também contribuiu pouco para a identificação das citadas respostas. Em função disso, um estudo de caso foi realizado na etapa (d), visando facilitar a investigação da natureza das relações entre os riscos. Além disso, o estudo de caso contribuiu para a validação dos grupos de riscos trazendo evidências baseadas em um caso real, que confirmavam que os riscos pertencentes a um mesmo grupo possuíam relações relevantes entre si. Os estudos baseados em caso são amplamente usados para explicitar os motivos pelos quais as relações ocorrem dentro dos construtos, e isto contribui para a validade interna destes (Yin, 2003).

A etapa (a) durou aproximadamente dois meses. A etapa (b), envolvendo o desenvolvimento do questionário, envio e recebimento das respostas, demorou quatro meses. As etapas (c) e (d) tiveram uma duração de aproximadamente um mês.

\subsection{Identificação de riscos à IPE}

A revisão da literatura enfatizou a busca de artigos recentes (2000-2012) em periódicos internacionais de reconhecida relevância na área de gestão de operações. As pesquisas que versam sobre a aplicação de práticas isoladas (por exemplo, trabalho padronizado, células de manufatura, sistema kanban) foram descartadas, visto que provavelmente não refletiriam os riscos de um processo mais complexo de IPE. Tendo em vista enriquecer as perspectivas sobre o tema, houve preocupação em selecionar estudos com diferentes orientações metodológicas, como estudos de caso, surveys e revisões da literatura.

Na literatura pesquisada, os riscos no processo de IPE são designados por diferentes termos, tais como barreiras, dificuldades e fatores de impacto. Assim, foi necessário selecionar aqueles estudos cujos resultados fossem consistentes com a definição de risco proposta pelo PMI (Project Management Institute, 2004), já apresentada na seção 1. Por exemplo, a influência do tipo de processo (White \& Prybutok, 2001) ou idade da planta (Shah \& Ward, 2003) não foram considerados riscos, pois não constituíam em si incertezas. Essas são características inerentes à planta ou setor produtivo.

Uma lista com 18 riscos à IPE foi inicialmente elaborada. Tal lista foi posteriormente reduzida, visto que por vezes havia sobreposição entre os riscos, pois os autores usavam expressões diferentes para designar um mesmo assunto. Por exemplo, a aplicação de práticas lean isoladas, sem visão dos impactos sistêmicos de cada prática (Mathaisel, 2005), foi associada ao risco denominado falta de conhecimento técnico das áreas de apoio (Sim \& Rogers, 2008). Após esse refinamento, identificaram-se 14 riscos, sendo para cada um estabelecidas evidências, como mostra a Tabela 1. Os trabalhos consultados apresentaram somente riscos internos à empresa na IPE. Entretanto, riscos externos, como um desastre natural, uma greve geral ou mudança na legislação, também podem ter impacto na IPE.

\subsection{Concepção e aplicação de questionários para avaliar percepções acerca da probabilidade e impacto de cada risco}

A lista de riscos desenvolvida a partir da revisão bibliográfica foi utilizada como base para uma survey, destinada a coletar dados para classificar os riscos na IPE. A amostra foi constituída por pessoas vinculadas a empresas que atendessem aos seguintes critérios: (a) existência de iniciativas de IPE; (b) empresas em diferentes estágios de IPE, pois alguns riscos podem aparecer em momentos específicos do processo; (c) empresas de diferentes setores, pois existe a necessidade de compreender as manifestações dos riscos em cada setor; (d) empresas localizadas em uma região específica do país, nesse caso a região Sul, para reduzir os efeitos do ambiente externo (por 
Tabela 1. Riscos à IPE.

R1 - Desmotivação dos envolvidos após as primeiras mudanças

Evidências: desinteresse das pessoas em participar de atividades ligadas ao processo após alguns meses ou anos do início do processo.

R2 - Falta de conhecimento das áreas de apoio (Engenharia, Manutenção e áreas dedicadas ao lean) para orientar o andamento da IPE

Evidências: aplicação de práticas lean isoladas, sem integração ou visão dos impactos sistêmicos da implantação de cada prática. Insegurança e receio das áreas de apoio em orientar e aplicar práticas lean. Dificuldade em identificar quais as práticas necessárias e como devem ser implantadas.

R3 - Falta de recursos humanos e ou financeiros destinados ao processo

Evidências: não destinar tempo suficiente para as pessoas realizarem atividades de treinamento e aplicação das práticas lean. Não destinar recursos financeiros suficientes para treinar os funcionários em lean.

R4 - Falta de clareza na comunicação para todos os funcionários sobre o início e o progresso da implantação Evidências: não divulgar por meios oficiais os resultados alcançados, as atividades realizadas, os participantes do processo e os objetivos.

R5 - Dificuldades de comprovar o retorno financeiro das ações realizadas

Evidências: apego aos indicadores tradicionais que não apontam resultados como atendimento ao cliente, estoque

em excesso, área liberada, entre outros. Priorizar ações de impacto em indicadores de curto prazo, sem valorizar melhorias qualitativas ou de maior resultado a médio e longo prazo.

R6 - Falta de apoio da média gerência

Evidências: a média gerência não cobrar prazos e resultados do processo, não disponibilizar tempo para esclarecer dúvidas e resolver problemas de implantação, ou não ter comprometimento com a aplicação das práticas lean e de seus benefícios

R7 - Falta de apoio da alta direção

Evidências: a alta direção não controlar e auxiliar no andamento do processo, não vincular as ações às metas e aos objetivos do negócio, ou priorizar outras ações em detrimento daquelas que envolvam a IPE.

R8 - 0 nível operacional não apoiar a implantação

Evidências: operadores ou supervisores sem o interesse em aplicar ou usar as práticas lean. Receio dos operadores e supervisores em manter o emprego, em função de a IPE levar ao uso de menos recursos (operadores, estoque ou máquinas) para atingir as metas

R9 - Insegurança dos operadores na realização de novas atribuições

Evidências: os supervisores e áreas de apoio não apoiarem os operadores na realização de melhorias, no trabalho em equipe, na padronização, no uso de quadros de acompanhamento da produção, ao chamado da cadeia de ajuda e na parada da produção. Os supervisores não respeitarem as ideias e sugestões de melhorias provenientes dos operadores. Não capacitarem os operadores para estas novas atribuições.

R10 - A demissão de operadores em função do excesso de mão de obra gerado pelas melhorias implantadas Evidências: após a realização de atividades de melhorias (ou kaizens) na fábrica, demitir os operadores que não são mais necessários pelos ganhos de produtividade alcançados. Demitir operadores de acordo com a oscilação da demanda.

R11 - Os operadores não se sentirem responsáveis pelo uso das práticas lean e pela solução de problemas Evidências: não envolver os operadores na realização de melhorias na fábrica e na implantação de práticas de PE. Falta de participação dos operadores no processo de implantação.

R12 - Os gerentes e diretores não terem o conhecimento suficiente sobre o lean

Evidências: dificuldade da alta e média gerência em estabelecer objetivos para implantar, acompanhar e garantir a utilização dos princípios e práticas lean. A alta e média gerência não guiar a IPE.

R13 - Não sustentar as melhorias a médio e longo prazo

Evidências: melhorias realizadas na aplicação de práticas ou solução de problemas que acabam retornando ao estado

original após alguns meses. Não há um acompanhamento por meio auditorias, nem padronização das melhorias.

R14 - Dificuldades na gestão do processo de implantação

Evidências: os responsáveis pelo processo não cobrarem prazos e acompanharem as atividades previstas. Falta de auditorias no andamento do processo. Falta de comprometimento com os prazos e objetivos do processo.

$3,4,5,7,8,9,13,14$

*Referências: 1- Black (2007), 2 - Mathaisel (2005), 3 - Bhasin (2012), 4 - Achanga et al. (2006), 5 - Sim \& Rogers (2008), 6 - Piercy \& Rich (2009), 7 - Papadopoulou \& Özbayrak (2005), 8 - Panizzolo et al. (2012), 9 - Sriparavastu \& Gupta (1997), 10 - Shah \& Ward (2003); 11 - Bayo-Moriones et al. (2008), 12 - Farris et al. (2009), 13 - Emiliani \& Stec (2005), 14 - Turesky \& Connell (2010). Fonte: Elaborado pelos autores.

exemplo, infraestrutura pública de transporte, perfil e disponibilidade de mão de obra), visto que esse seria relativamente homogêneo no âmbito da amostra.

Em relação aos critérios usados para selecionar os respondentes da survey em cada empresa, foram priorizados aqueles que haviam participado, entre os anos de 2008 e 2010, de cursos sobre PE oferecidos pela instituição responsável por essa pesquisa ou por uma instituição que atua desde 1998 na disseminação do sistema enxuto em âmbito nacional. A instituição responsável pela pesquisa era a única Universidade na época a oferecer cursos sobre o assunto na região Sul, abertos ao público em geral e divulgados em grandes mídias. Dessa forma, a amostra envolveria pessoas com conhecimento técnico para responder à survey, somando um total de 305 candidatos. A escolha não randômica de empresas para surveys e a busca por empresas já conhecidas dos pesquisadores são 
procedimentos usados em outros trabalhos sobre lean, tais como aqueles realizados por Farris et al. (2009) e Saurin et al. (2010).

0 questionário foi dividido em duas partes. $\mathrm{Na}$ primeira, para cada risco, o respondente deveria indicar o seu impacto e probabilidade. Havia uma escala de "0" a "5", com seis pontos, em que o valor " 0 " correspondia ao impacto ou à probabilidade inexistentes e o valor " 5 " correspondia a um impacto ou probabilidade muito alta. Assim, a primeira parte do questionário envolvia 28 perguntas ( 2 perguntas para cada um dos 14 riscos). A segunda parte do questionário continha 9 perguntas sobre o perfil dos respondentes e da empresa. A Figura 1 apresenta um exemplo da primeira parte do questionário.

Realizou-se um pré-teste com quatro integrantes da amostra e algumas perguntas do questionário foram reformuladas. 0 pré-teste é geralmente feito em uma pequena amostra e visa reduzir problemas referentes ao conteúdo, formato e clareza das perguntas e opções de resposta (Malhotra, 2004).

Com base nas sugestões de Umbach (2004) para planejar e executar web surveys, adotaram-se os seguintes procedimentos visando aumentar a taxa de respostas válidas: (a) os respondentes tinham duas opções de plataformas para preencher o questionário (uma planilha de Excel e uma página na Internet); (b) a interface gráfica da página na Internet deveria primar pela simplicidade e facilidade de uso; (c) o questionário deveria ser enviado duas vezes para toda a amostra, além de haver um terceiro lembrete por meio de contato telefônico; (d) todos os componentes da amostra deveriam ter e-mail, já que esse seria o principal canal de contato entre a equipe de pesquisa e os respondentes; (e) o conteúdo do e-mail deveria: descrever o objetivo da pesquisa, indicar qual o grupo de pessoas que estavam selecionadas para a pesquisa; informar uma data-limite para retorno dos questionários; informar que o tempo estimado para responder ao questionário era em torno de 10 a 20 minutos; informar que, caso fosse de seu interesse, o respondente poderia receber um retorno com o resultado da pesquisa.

Dentre os 305 participantes inicialmente selecionados, 12 deles não foram contatados, pois os endereços de e-mail estavam inválidos, e 3 pessoas responderam que não se consideravam capazes de responder ao questionário, pois não haviam participado de uma IPE. Ao todo, 57 respondentes preencheram o questionário, correspondendo a um percentual de 19\% dos contatos válidos. Este é um percentual menor em comparação a outras surveys sobre IPE em que os questionários foram enviados via postal (por exemplo, a taxa de retorno foi de 37\% no estudo de Moreira \& Alvez, 2008), porém o percentual está próximo da taxa média de respostas em pesquisas realizadas por e-mail, que é de 15\% (Malhotra, 2004).

\subsection{Análise dos resultados da aplicação do questionário}

A classificação dos riscos foi feita a partir de uma AFE, realizada no software SPSS versão 18. 0 resultado da multiplicação entre o impacto e a probabilidade foi usado para definir o valor final de cada risco, na visão de cada respondente. A AFE tem como objetivo identificar uma pequena quantidade de temas, dimensões, componentes ou construtos que representam as relações de um grande número de variáveis (Meyers et al., 2006). Isto é feito a partir da identificação de um grupo de variáveis correlacionadas, chamado de fator (Hair et al., 2006). A AFE é uma técnica de redução de dados amplamente utilizada nas ciências humanas para organizar um grupo de medidas em subgrupos ou categorias (Meyers et al.,

\section{1 - Desmotivação dos envolvidos após as primeiras mudanças \\ Exemplo: desinteresse das pessoas em participar de atividades ligadas ao projeto após alguns meses ou anos do início do projeto.

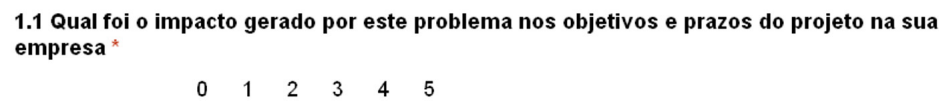

Figura 1. Exemplo da primeira parte do questionário. Fonte: Elaborado pelos autores. 
2006). Uma verificação da correlação direta de Pearson foi realizada antes da AFE.

0 tamanho da amostra necessária para viabilizar uma AFE depende da magnitude da correlação entre a população e o número de construtos a serem analisados (Tabachnick \& Fidell, 2001). Segundo esses autores, se a correlação for forte e existirem poucos e distintos construtos, mesmo uma pequena amostra já pode ser considerada adequada. Hair et al. (2006) recomendam que uma AFE seja baseada em no mínimo 50 observações. A AFE foi feita com uma extração por componentes principais com o método de rotação ortogonal Varimax. 0 número de grupos foi determinado considerando autovalor superior a 1,0 (Field, 2005), fato que resultou na definição de três grupos.

0 teste de Kaiser-Meyer-Olkin (KMO) foi usado para validar o grau de suscetibilidade ou o ajuste dos dados do questionário à AFE, pois ele indica o nível de confiança dos dados para o uso da AFE. 0 teste $\mathrm{KMO}$ obteve resultado 0,778 , acima do valor de 0,7 sugerido por Meyers et al. (2006). A significância obtida foi menor que 0,001 , indicando que os dados eram adequados para a utilização da AFE. 0 autovalor para o G1 $(5,338), \mathrm{G} 2(1,984)$ e G3 $(1,494)$ somou um percentual de variância total explicada acumulada de $62,97 \%$. Uma solução robusta para os agrupamentos criados pela AFE deve representar um valor acima de 50\% da variância total (Tabachnick \& Fidell, 2001).

A validação dos construtos foi feita através do alfa de Cronbach (Cronbach, 1951). Segundo Nunnally (1978), um alfa superior a 0,6 é suficiente para indicar que as escalas e os agrupamentos são válidos. Os agrupamentos feitos pela AFE apresentaram valores de alfa de 0,784, 0,723 e 0,816.

\subsection{Estudo de caso para identificação das relações entre os riscos em um contexto real}

A empresa $A B C$ fabrica semieixos automotivos e foi escolhida para o estudo de caso em função dos seguintes motivos: (a) três respondentes do questionário trabalhavam nessa empresa; (b) ser fornecedora da Toyota, que é reconhecida por realizar esforços de IPE ao longo de toda a cadeia de suprimentos (Panizzolo, 1998); (c) possuir experiência na IPE, mantendo uma estrutura dedicada com esforços contínuos nesse sentido desde 2004. Também vale salientar que os autores mantêm uma relação de parceria acadêmica com a empresa há vários anos (por exemplo, funcionários da empresa realizando cursos de pós-graduação na instituição pesquisadora, participação de membros da empresa em um grupo de troca de experiências em IPE, pesquisas realizadas por mestrandos da instituição na empresa), sendo que nos quatro anos que antecederam a pesquisa, os autores visitaram diversas vezes a empresa.

As principais fontes de evidências usadas no estudo foram entrevistas semiestruturadas, observações das atividades produtivas, análise de documentos (por exemplo, apresentações sobre as melhorias executadas e sobre o sistema de produção da empresa) e o desenvolvimento de três Mapas de Fluxo de Valor (MFV). 0 primeiro MFV foi feito durante um workshop promovido por funcionários da empresa e pelos autores em 2009, e os outros dois realizados durante disciplinas de Especialização em Engenharia de Produção oferecidas pela instituição de ensino dos autores durante os anos de 2011 e 2012. Os dados coletados para elaborar os MFV contribuíram para a compreensão do uso de práticas lean na empresa.

A escolha dos entrevistados levou em consideração a busca de perspectivas de diferentes pessoas envolvidas na IPE. Os entrevistados foram um analista de processo, cujo cargo na empresa era denominado especialista lean, um supervisor de produção e um gerente industrial. Cada entrevista durou em média duas horas.

As entrevistas foram realizadas com o suporte de um roteiro com 22 questões, divididas em dois grupos: (a) percepções acerca do planejamento do processo de IPE (por exemplo, atividades, quem estava envolvido, a duração, método e práticas aplicadas); (b) percepções sobre as principais dificuldades, riscos e medidas de controle. Em relação às observações, essas foram realizadas durante os MFV e em uma caminhada pelo chão de fábrica com acompanhamento de um dos entrevistados, quando houve a oportunidade da ilustração do uso de algumas práticas e princípios do sistema lean.

\section{Resultados}

\subsection{Caracterização da amostra: perfil dos respondentes}

A Tabela 2 apresenta o cargo dos respondentes e o setor da empresa ao qual eles estavam formalmente vinculados. Em relação ao cargo, percebe-se a predominância de cargos operacionais, como analistas e assistentes (33\%), e da baixa gerência, tais como supervisores e coordenadores (33\%). Os cargos de gerente (13\%) e diretor (7\%) foram considerados como média ou alta gerência. Este resultado indica que a amostra possuiu variedade em relação aos níveis hierárquicos e permitiu múltiplos pontos de vista em relação aos riscos envolvidos na $\mathrm{IPE}$, fator importante para investigar temas complexos em geral (Farris et al., 2009).

Havia uma pergunta sobre a experiência dos respondentes na IPE. A Tabela 3 mostra o resultado 
desta pergunta, à qual 86\% dos respondentes afirmaram ter experiência prática em lean por um período médio de 2,4 anos. Essa experiência corresponde ao envolvimento direto na aplicação das práticas lean.

Dessa forma, o perfil predominante da amostra foi de analistas e supervisores que possuem experiência prática e conhecimento teórico em IPE. Em relação à experiência prática, uma grande parcela dos respondentes (46\%) possui até dois anos atuando na implantação de práticas lean. Vale salientar a presença de um percentual razoável de respondentes (23\%) com mais de quatro anos de experiência direta na IPE.

\subsection{Caracterização da amostra: perfil das empresas}

0 critério de classificação do porte da empresa foi o utilizado pelo Sebrae e pelo IBGE (Serviço Brasileiro de Apoio às Micro e Pequenas Empresas, 2011).
Os resultados demonstram que 65\% das empresas eram de grande porte e $33 \%$ de médio porte, com apenas 2\% (uma empresa) de pequeno porte. Dentre as principais dificuldades para a IPE em pequenas empresas, Golhar et al. (1990) citam a falta de qualidade dos fornecedores e os custos elevados, principalmente com treinamentos e consultorias. Achanga et al. (2006), com base em múltiplos estudos de caso realizados com pequenas empresas, também identificaram a limitação financeira como uma dificuldade, embora apontem a falta de capacidade técnica e gerencial dos líderes como um risco ainda mais importante. De fato, raros são os relatos acerca do uso do sistema lean em pequenas empresas (Achanga et al., 2006; Womack \& Jones, 1996).

Em relação aos setores produtivos, pode-se identificar uma presença forte do setor de autopeças, com 28\% (Tabela 4). 0 próprio termo lean teve sua origem em estudos realizados na cadeia automotiva

Tabela 2. Cargo dos respondentes.

\begin{tabular}{lcc}
\multicolumn{1}{c}{ Cargo } & $\begin{array}{c}\text { Número de respondentes } \\
\text { Total }=57 \text { pessoas }\end{array}$ & Percentual (\%) \\
\hline Analista ou Assistente & 18 & 33 \\
Supervisor ou Coordenador & 18 & 33 \\
Gerente & 7 & 13 \\
Diretor & 4 & 7 \\
Estagiário ou Trainee & 4 & 7 \\
Consultor & 3 & 5 \\
Não responderam & 3 & \\
\hline
\end{tabular}

Fonte: Elaborado pelos autores.

Tabela 3. Experiência prática em IPE.

\begin{tabular}{lcc}
\multicolumn{1}{c}{ Experiência prática em PE } & Número de respondentes \\
Total $=57$ pessoas & Percentual (\%) \\
\hline Mais de 4 anos & 13 & 23 \\
de 2 a 4 anos & 10 & 18 \\
de 1 a 2 anos & 16 & 28 \\
Menos de 1 ano & 10 & 18 \\
Sem experiência & 8 & 14 \\
\hline
\end{tabular}

Fonte: Elaborado pelos autores.

Tabela 4. Setor das empresas.

\begin{tabular}{|c|c|c|}
\hline Setores & $\begin{array}{l}\text { Número de empresas } \\
\qquad N=39\end{array}$ & Percentual $(\%)$ \\
\hline Autopeças & 11 & 28 \\
\hline Bens de Consumo não duráveis & 4 & 10 \\
\hline Materiais de Construção & 4 & 10 \\
\hline Montadoras de Máquinas e Equipamentos & 3 & 8 \\
\hline Agroindustrial & 3 & 8 \\
\hline Vestuário e Acessórios & 2 & 5 \\
\hline Fabricação de Móveis & 2 & 5 \\
\hline Saúde & 2 & 5 \\
\hline Consultoria & 1 & 3 \\
\hline $\begin{array}{l}\text { Outros: Aeronáutico, Eletrodomésticos, Eletrônicos, Logística e Transporte, } \\
\text { Metalomecânica, Químicos e Plásticos, Siderurgia }\end{array}$ & $\begin{array}{c}7 \\
\text { (1 cada) }\end{array}$ & $\begin{array}{c}21 \\
(3 \% \text { cada })\end{array}$ \\
\hline
\end{tabular}

Fonte: Elaborado pelos autores. 
(Krafcit, 1988), e a difusão ocorreu não apenas por trabalhos realizados na Toyota (Lander \& Liker, 2007), mas também em outras montadoras, como Ford e GM (Bayou \& Korvin, 2008). Apesar disso, vale enfatizar que a maior parte das empresas não pertence ao setor automobilístico (72\%) e estão pulverizadas em outros setores. A IPE em diferentes setores requer o enfoque nos princípios do sistema lean, em vez do enfoque em copiar as práticas enxutas (Womack \& Jones, 1996).

A Tabela 5 indica há quantos anos a empresa vem mantendo um processo formal de IPE. É possível identificar quatro grupos de empresas: (a) aquelas que mantêm processos formais de IPE há mais de quatro anos (31\%); (b) aquelas que não completaram dois anos do início da implantação (41\%); (c) aquelas que não iniciaram um processo formal de IPE (18\%); (d) um pequeno grupo de empresas (10\%) que se manteve no período intermediário, de dois a quatro anos. Vale salientar que o fato de algumas empresas não apresentarem um processo formal de IPE não significa que elas não estejam mantendo esforços informais ou pontuais de aplicação das práticas lean.

Uma vez que os dados foram coletados no final de 2010, pode-se perceber que a maior parte das empresas iniciou o processo de implantação em 2009, ou seja, imediatamente após ou em meio a um período de crise no mercado mundial. Esse fato é consistente com a percepção de que uma crise é um estímulo para a IPE, pois, a partir da necessidade, as empresas se veem compelidas a melhorar seu desempenho (Womack \& Jones, 1996).

Desse modo, o perfil das empresas em que trabalham os respondentes é predominantemente de grande porte, que atuam em diferentes setores, com ligeira concentração no setor de autopeças. Além disso, a maioria delas ainda não completou dois anos de processos formais de IPE, estando, possivelmente, em uma fase de conhecimento e experimentação das práticas.

\subsection{Classificação em grupos}

A Tabela 6 apresenta as correlações de Pearson diretas entre os riscos, enquanto a Tabela 7 mostra as cargas fatoriais de cada risco e os grupos resultantes da AFE.

Os riscos R2 e R14 foram os únicos que apresentaram uma carga fatorial inferior a 0,5, o que indica uma relação similar com dois dos três grupos. Esses dois riscos foram testados em agrupamentos diferentes, optando-se por aquele que resultava em maior homogeneidade com base no alfa de Cronbach, para

Tabela 5. Há quantos anos um processo formal de IPE está em curso.

\begin{tabular}{lcc}
\hline \multicolumn{1}{c}{ Anos de processo formal de PE } & $\begin{array}{c}\text { Número de empresas } \\
\text { N }=39\end{array}$ & Percentual (\%) \\
\hline Mais de 6 anos & 3 & 8 \\
de 4 a 6 anos & 9 & 23 \\
de 2 a 4 anos & 4 & 10 \\
de 1 a 2 anos & 9 & 23 \\
até 1 ano & 7 & 18 \\
Não há um processo formal & 7 & 18 \\
\hline
\end{tabular}

Fonte: Elaborado pelos autores.

Tabela 6. Correlações de Pearson entre os riscos.

\begin{tabular}{|c|c|c|c|c|c|c|c|c|c|c|c|c|c|c|}
\hline Risco & R1 & $\mathrm{R} 2$ & R3 & R4 & R5 & R6 & R7 & R8 & R9 & R10 & R11 & $\mathrm{R} 12$ & R13 & R14 \\
\hline $\mathrm{R} 1$ & 1 & $0,309^{*}$ & $0,266^{*}$ & $0,445^{* *}$ & $0,353^{* * *}$ & 0,246 & $0,297^{*}$ & $0,450^{* * *}$ & 0,170 & 0,325 & $0,411^{* *}$ & $0,438^{* *}$ & $0,326^{*}$ & $0,415^{* *}$ \\
\hline $\mathrm{R} 2$ & $0,309^{*}$ & 1 & $0,268^{*}$ & 0,246 & 0,14 & $0,394^{* *}$ & $0,372^{* *}$ & $0,356^{* * *}$ & $0,307^{*}$ & 0,18 & $0,439^{* *}$ & $0,415^{* *}$ & $0,289^{*}$ & $0,462^{* *}$ \\
\hline R3 & $0,266^{*}$ & $0,268^{*}$ & 1 & 0,088 & 0,215 & $0,455^{* * *}$ & $0,539^{* *}$ & $0,297^{*}$ & $0,379^{* * *}$ & $0,469^{* *}$ & 0,21 & $0,273^{*}$ & 0,239 & 0,233 \\
\hline R4 & $0,445^{* *}$ & 0,246 & 0,088 & 1 & $0,343^{* *}$ & $0,379^{* *}$ & $0,312^{*}$ & $0,422^{* * *}$ & $0,293^{*}$ & 0,21 & $0,405^{* *}$ & $0,485^{* *}$ & $0,304^{*}$ & $0,387^{* * *}$ \\
\hline R5 & $0,353^{* * *}$ & 0,14 & 0,215 & $0,343^{* * *}$ & 1 & $0,296^{*}$ & 0,184 & 0,09 & 0,144 & 0,2 & 0,188 & 0,2 & 0,227 & 0,235 \\
\hline R6 & 0,246 & $0,394^{* *}$ & $0,455^{* *}$ & $0,379^{* *}$ & $0,296^{*}$ & 1 & $0,789^{* *}$ & 0,242 & 0,247 & 0,143 & 0,146 & $0,385^{* *}$ & $0,331^{*}$ & $0,374^{* *}$ \\
\hline R7 & $0,297^{*}$ & $0,372^{* *}$ & $0,539^{* * *}$ & $0,312^{*}$ & 0,184 & $0,789^{* *}$ & 1 & $0,367^{* * *}$ & $0,292^{*}$ & $0,272^{*}$ & 0,146 & $0,491^{* *}$ & $0,411^{* *}$ & $0,425^{* * *}$ \\
\hline R8 & $0,450^{* *}$ & $0,356^{* * *}$ & $0,297^{*}$ & $0,422^{* *}$ & 0,09 & 0,242 & $0,367^{* *}$ & 1 & $0,373^{* *}$ & $0,481^{*}$ & $0,366^{* *}$ & 0,178 & $0,598^{* *}$ & \\
\hline R9 & 0,17 & $0,307^{*}$ & $0,379^{* *}$ & $0,293^{*}$ & 0,144 & 0,247 & $0,292^{*}$ & $0,311^{*}$ & 1 & $0,389^{* * *}$ & $0,447^{* *}$ & $0,309^{*}$ & $0,264^{*}$ & $0,616^{* *}$ \\
\hline R10 & 0,325 & 0,18 & $0,469^{* * *}$ & 0,21 & 0,2 & 0,143 & $0,272^{*}$ & $0,373^{* *}$ & $0,389^{* *}$ & 1 & $0,375^{* *}$ & $0,273^{*}$ & 0,116 & $0,275^{*}$ \\
\hline R11 & $0,411^{* *}$ & $0,439^{* *}$ & 0,21 & $0,405^{* *}$ & 0,188 & 0,146 & 0,146 & $0,481^{*}$ & $0,447^{* *}$ & $0,375^{* *}$ & 1 & $0,444^{* *}$ & 0,238 & $0,481^{* * *}$ \\
\hline $\mathrm{R} 12$ & $0,438^{* *}$ & $0,415^{* * *}$ & $0,273^{*}$ & $0,485^{* *}$ & 0,2 & $0,385^{* *}$ & $0,491^{* *}$ & $0,366^{* *}$ & $0,309^{*}$ & $0,273^{*}$ & $0,444^{* * *}$ & 1 & $0,397^{* *}$ & $0,434^{* * *}$ \\
\hline R13 & $0,326^{*}$ & $0,289^{*}$ & 0,239 & $0,304^{*}$ & 0,227 & $0,331^{*}$ & $0,411^{* * *}$ & 0,178 & $0,264^{*}$ & 0,116 & 0,238 & $0,397^{* * *}$ & 1 & $0,432^{* *}$ \\
\hline R14 & $0,415^{* *}$ & $0,462^{* * *}$ & 0,233 & $0,387^{* *}$ & 0,235 & $0,374^{* * *}$ & $0,425^{* *}$ & $0,598^{* * *}$ & $0,616^{* *}$ & $0,275^{*}$ & $0,481^{* *}$ & $0,434^{* *}$ & $0,432^{* *}$ & 1 \\
\hline
\end{tabular}

${ }^{*}$ Correlação significante para o nível 0.05. **Correlação significante para o nível 0.01. Fonte: Elaborado pelos autores. 
os dois agrupamentos simultaneamente. Por exemplo, foi calculado o alfa com o R14 pertencendo ao G1 e ao G3. Quando ocorreu a alocação desse risco no G3, o grau de homogeneidade do G1 aumentou, mas o alfa do G3 reduziu para um valor abaixo de 0,7 , ou seja, menor que o resultado considerado adequado (Nunnally, 1978). Por isso, em função de trazerem maior homogeneidade aos agrupamentos, o R14 e o R2 foram enquadrados no G1.

Tendo em vista facilitar a identificação dos grupos, estabeleceu-se uma denominação para cada

Tabela 7. Cargas fatoriais e agrupamentos dos riscos na IPE*.

\begin{tabular}{cccc}
$\begin{array}{c}\text { Riscos \Grupo } \\
\text { (Alfa de Cronbach) }\end{array}$ & $\begin{array}{c}\text { G 1 } \\
\text { Áreas de Apoio } \\
(0,784)\end{array}$ & $\begin{array}{c}\text { G 2 } \\
\text { Gerência } \\
(0,816)\end{array}$ & $\begin{array}{c}\text { G 3 } \\
\text { Operacional } \\
(0,723)\end{array}$ \\
\hline R1 & 0,604 & 0,064 & 0,378 \\
R2 & 0,400 & 0,287 & 0,364 \\
R3 & $-0,082$ & 0,725 & 0,396 \\
R4 & 0,719 & 0,044 & 0,269 \\
R5 & 0,624 & 0,154 & $-0,093$ \\
R6 & 0,384 & 0,803 & 0,022 \\
R7 & 0,294 & 0,848 & 0,167 \\
R8 & 0,318 & 0,093 & 0,668 \\
R9 & 0,100 & 0,241 & 0,669 \\
R10 & $-0,139$ & 0,247 & 0,728 \\
R11 & 0,393 & $-0,111$ & 0,713 \\
R12 & 0,557 & 0,285 & 0,362 \\
R13 & 0,518 & 0,348 & 0,110 \\
R14 & 0,465 & 0,205 & 0,490 \\
\hline Fa & & & \\
\hline
\end{tabular}

*Nota: Os valores em negrito indicam em qual o agrupamento o risco foi alocado. Fonte: Elaborado pelos autores. um, com base na natureza dos seus componentes. 0 nível hierárquico demonstrou ser o diferencial adequado para distinguir cada classificação (Tabela 8). A Tabela 8 apresenta também a média de cada risco individualmente e de cada grupo.

Sete riscos foram associados ao agrupamento denominado “Áreas de Apoio". Os funcionários vinculados às áreas de apoio são geralmente os responsáveis diretos pela implantação e sustentação das práticas lean. As áreas de apoio exercem um papel essencial de capacitar a todos no uso das práticas lean (Motwani, 2003), assim, elas necessitam de competência técnica (Emiliani \& Stec, 2005) e gerencial (Mathaisel, 2005).

0 R13 (não sustentar as melhorias a médio e longo prazo) apresentou o maior índice $(3,89)$ dentre os 14 riscos. As características das empresas pesquisadas podem explicar a importância atribuída ao R13, visto que a média de idade dos processos formais de IPE foi de 2,5 anos, tempo que pode ser suficiente para as empresas perceberem a dificuldade de manter os ganhos obtidos nas fases iniciais de implantação.

Vale destacar a presença do R12 (os gerentes e diretores não terem o conhecimento suficiente sobre a IPE) em um grupo de riscos denominado "Áreas de Apoio", em vez do grupo de riscos denominado "Alta e Média Gerência”. Uma possível interpretação para essa classificação é a de que o R12, em termos operacionais, torna-se visível e é sentido pelas áreas de apoio. Nessa área, geralmente há o grupo de pessoas com maior conhecimento na IPE, as quais percebem e devem lidar com decisões de gerentes e diretores que por vezes são conflitantes com os objetivos da PE. A Tabela 6 mostra que a carga fatorial desse risco para o G1 $(0,557)$ foi maior que o dobro da carga fatorial para o $\mathrm{G} 2(0,285)$.

0 agrupamento denominado "Alta e Média Gerência” apresentou apenas três riscos. Segundo

Tabela 8. Classificação de riscos na implantação de PE.

\begin{tabular}{|c|c|c|}
\hline Grupo & Índice & Risco \\
\hline \multirow{7}{*}{ G1: Áreas de apoio - 3,46 } & 3,44 & R1 - Desmotivação dos envolvidos após as primeiras mudanças. \\
\hline & 3,50 & $\begin{array}{l}\text { R2 - Falta de conhecimento das áreas de apoio (Engenharia, Manutenção e áreas dedicadas à PE) para } \\
\text { orientar o andamento da IPE. }\end{array}$ \\
\hline & 3,09 & R4 - Falta de clareza na comunicação para toda a empresa sobre o início e o progresso da implantação. \\
\hline & 3,66 & R5 - Dificuldades de enxergar o retorno financeiro nas ações realizadas. \\
\hline & 3,19 & R12 - Os gerentes e diretores não terem o conhecimento suficiente sobre a PE. \\
\hline & 3,89 & R13 - Não sustentar as melhorias a médio e longo prazo. \\
\hline & 3,35 & R14 - Dificuldades na gestão do processo de implantação. \\
\hline \multirow{3}{*}{$\begin{array}{l}\text { G2 - Alta e média } \\
\text { gerência - } 3,25\end{array}$} & 3,30 & R3 - Falta de recursos (humanos e ou financeiros) destinados ao processo. \\
\hline & 3,31 & R6 - Falta de apoio da média gerência. \\
\hline & 3,16 & R7 - Falta de apoio da alta direção. \\
\hline \multirow{4}{*}{$\begin{array}{l}\text { G3 - Nível operacional } \\
-2,96\end{array}$} & 3,31 & R8 - 0 nível operacional não apoiar a implantação. \\
\hline & 3,17 & R9 - Insegurança dos operadores na realização de novas atribuições. \\
\hline & 2,30 & R10 - A demissão de operadores em função do excesso de mão de obra gerado pelas melhorias implantadas. \\
\hline & 3,01 & R11 - Os operadores não se sentirem responsáveis pelo uso das práticas de PE e pela solução de problemas. \\
\hline
\end{tabular}

Fonte: Elaborado pelos autores. 
Sim \& Rogers (2008), esses níveis geralmente falham em comunicar, apoiar e guiar o processo de IPE. Quanto aos riscos associados ao agrupamento "Nível Operacional”, o R10 (a demissão de operadores em função do excesso de mão de obra gerado pelas melhorias implantadas) apresentou o menor valor dentre os 14 riscos. Esse resultado é positivo, uma vez que as demissões causadas por aumentos de produtividade são comuns e reduzem o incentivo dos operadores em contribuir para melhorias, reduzem o ritmo da implantação e violam o princípio da PE de respeito às pessoas (Emiliani \& Stec, 2005).

\subsection{Processo de IPE na empresa $A B C$}

A empresa $A B C$ é uma multinacional que atua no ramo de componentes e sistemas de transmissão para veículos leves e pesados. Os seus clientes são montadoras de veículos e o mercado de reposição, tanto no âmbito nacional como internacional. A empresa possui duas plantas no Brasil, sendo que a planta visitada está localizada no Sul do país. Esta planta, no período da pesquisa, contava com cerca de 700 funcionários. Os processos de fabricação desta planta envolvem forjarias, usinagens e montagens.

A IPE iniciou em 2004 nesta planta, motivada pela necessidade de reduzir custos e melhorar os índices de qualidade. 0 maior problema de qualidade na época era a omissão de alguma operação de fabricação, a qual geralmente só era identificada nas inspeções ao final do fluxo de valor. 0 arranjo físico por processos, até então existente, contribuía para essas omissões, pois o fluxo confuso e com múltiplas possibilidades induzia a erros na sequência das operações.

Assim, durante dois anos, foram realizadas mudanças organizacionais e no arranjo físico, visando à criação de células de manufatura. As células são amplamente reconhecidas por terem objetivos compatíveis com o princípio do fluxo contínuo (Womack \& Jones, 1996). Contudo, a equipe envolvida nessa mudança não teve apoio técnico ou treinamento adequado nesse período, o que dificultou em muito os avanços da IPE.

Em 2006, com a troca da diretoria da empresa no Brasil, o apoio da alta administração se tornou mais incisivo. Por exemplo, um grupo de 20 pessoas foi treinado em algumas práticas lean, e algumas dessas pessoas se tornaram dedicadas à IPE. 0 mapeamento de fluxo de valor, a troca rápida de ferramentas, manutenção preventiva, trabalho padronizado e poka-yokes são alguns exemplos de práticas aplicadas. Nessa fase, algumas das dificuldades enfrentadas foram a resistência dos operadores e de supervisores às novas atribuições criadas pelas práticas lean, bem como a ênfase nos indicadores de curto prazo, tais como payback e retorno sobre o investimento de cada kaizen. Tal ênfase decorria da falta de compreensão da diretoria sobre o sistema lean, além do uso simultâneo, pela empresa, de outros métodos de melhoria, como o Seis Sigma. Esses métodos priorizavam as ações pelo retorno financeiro em curto prazo e utilizavam métodos estatísticos complexos que dificultam a compreensão de todos acerca da razão pelas quais certas mudanças eram implantadas e outras não, além de que dificilmente resultavam em mudanças operacionais.

Um ex-executivo da Toyota assumiu um cargo na alta administração da empresa em nível mundial em 2008 e, no ano seguinte, a IPE adquiriu maior velocidade. Este executivo coordenou a criação de um sistema de produção, padronizado e definido pela matriz, fazendo com que a aplicação das práticas lean nas áreas fabris e administrativas se tornasse obrigatória para todas as plantas da empresa no mundo. A responsabilidade pela implantação e sustentação das práticas lean passou a ser exclusivamente dos próprios supervisores e gerentes de produção, e não mais da área de engenharia, como era entre $2004 \mathrm{e}$ 2006, ou da área de melhoria contínua, como era entre 2006 e 2008. Essa alteração na responsabilidade foi importante pelo fato de que, anteriormente, a IPE era vista pelos supervisores e gerentes de produção como um projeto das áreas de apoio e engenharia, ou seja, eram tarefas fora de sua rotina diária. Segundo um dos entrevistados, que atuava como analista de processo nesse período, para a maioria dos supervisores de produção "o lean era mais uma coisa para fazer além do meu trabalho do dia a dia e não o modo de realizar as atividades do dia a dia". Quando a responsabilidade sobre a aplicação das práticas lean foi transferida para os supervisores e gerentes de produção, tais tarefas passaram a fazer parte da rotina dessas pessoas. Nessa fase, foram identificados grandes avanços na utilização das práticas lean, fato vinculado a esse sistema de produção ter sido definido e aplicado a partir da alta administração.

\subsubsection{Riscos associados às áreas de apoio (G1)}

Algumas das relações entre os riscos do $\mathrm{G} 1$ foram evidenciadas na empresa ABC. Por exemplo, a relação entre o R12 (os gerentes e diretores não terem o conhecimento suficiente sobre a PE) e o R2 (falta de conhecimento das áreas de apoio para orientar o andamento da IPE) foi notória, principalmente entre 2004 e 2006. A falta de conhecimento de diretores e gerentes fez com que não houvesse indicadores de desempenho vinculados à IPE, além de não haver clareza acerca de quais princípios e práticas deveriam ser aplicados. Além disso, os gestores não identificaram 
as necessidades de capacitação técnica ou gerencial dos responsáveis pela IPE. Em função desse cenário, os responsáveis pelas áreas de apoio se sentiam inseguros em realizar as atividades vinculadas à IPE.

A falta de conhecimento dos gerentes e diretores também levava à dificuldade de medição do retorno financeiro das ações realizadas (R5). Em função disso, o direcionamento e priorização das ações enfatizava indicadores de retorno em curto prazo (ver seção 4.4), e/ou ligados à produção em massa, por exemplo, volume de produção e eficiência de equipamentos.

Como resultado, em algumas ocasiões a gerência exigia ações contrárias aos princípios lean. Por exemplo, o supervisor de produção relatou que "mais de uma vez a gerência exigiu o aumento de estoques e contratação de funcionários temporários para suprir uma determinada previsão de demanda futura (ações que geram as perdas de produção em excesso, estoque e transporte), em vez de buscar a redução de tempo de setup, melhorias de estabilidade ou de produtividade para garantir o atendimento ao cliente". Tais fatos também tiveram impacto no R14 (dificuldades na gestão do processo de implantação), pois muitas vezes os recursos previstos para ações vinculadas à IPE eram redirecionados a outros projetos em função de objetivos de curto prazo.

Por outro lado, o R14 tinha influência no R13 (não sustentar as melhorias a médio e longo prazo), pois a sustentação das melhorias se tornava cada vez mais difícil com as constantes mudanças de prioridades. Os funcionários do setor de melhoria contínua tinham o conhecimento sobre o enfoque sistêmico e de eliminação de perdas da IPE, mas se viam obrigados a seguir as diretrizes vindas da alta e média gerência.

Contudo, tais riscos se tornaram menores com a já citada entrada na empresa de um executivo corporativo em 2008, com profundo conhecimento e experiência em lean. A partir desse momento, desenvolveu-se um sistema de produção que definia com clareza as práticas a serem utilizadas, os principais indicadores e o papel de cada nível hierárquico na solução de problemas. Esse executivo acompanhou pessoalmente a implantação do sistema de produção, por meio de visitas às fábricas e tutoria aos envolvidos. Tal fato tornou o ritmo de IPE mais acelerado. A criação do sistema de produção corporativo, definição do papel de cada área e a influência desse executivo tiveram um impacto direto nos riscos deste grupo.

\subsubsection{Riscos associados à alta e média gerência (G2)}

Na empresa $A B C$, os riscos R6 (falta de apoio da média gerência) e R7 (falta de apoio da alta direção) demonstraram relação, principalmente durante os anos de 2004 a 2008. A diretoria afirmava que tinha interesse na IPE para a planta ganhar competitividade no mercado, porém não monitorava o desempenho do processo, além de não vincular a PE às metas do ano e aos objetivos do negócio. Uma vez que a direção não cobrava da média gerência um envolvimento com a PE, essa também não percebia a necessidade de acompanhar, priorizar as ações ou auxiliar no processo.

De outro lado, os riscos R6 e R7 não tiveram aparente impacto no R3 (falta de recursos humanos e ou financeiros destinados ao processo), pois o processo de IPE sempre foi apoiado com recursos humanos e financeiros adequados. A disponibilidade de recursos na $A B C$ pode ser explicada pelo fato de ser uma empresa multinacional de grande porte e do ramo automotivo, que sempre teve um incentivo corporativo e dos seus clientes para a IPE. Contudo, o impacto de R6 e R7 em R3 deve ser sempre considerado como uma possibilidade real, na medida em que a falta de apoio da alta e média gerência pode, por exemplo, restringir investimentos em treinamentos e não destinar o tempo necessário para os funcionários participarem nas atividades de melhoria.

\subsubsection{Riscos associados ao nível operacional (G3)}

Na empresa $A B C$, o risco R10 (demissão de operadores em função das melhorias decorrentes da PE) foi relevante, na medida em que a demissão de operadores causou um forte impacto negativo, em contraste com o resultado médio obtido na aplicação do questionário. 0 impacto do R10 no R8 (o nível operacional não apoiar a implantação) foi passível de verificação no início do processo, de 2004 a 2006. Desde o princípio, os operadores estavam receosos com as mudanças que a PE acarretaria no trabalho de cada um e isso piorou devido a um evento relatado. No ano de 2005, houve a mudança de um arranjo físico por processo para uma célula de manufatura, incluindo o rebalanceamento dos postos de trabalho. Naquela ocasião, foi possível reduzir a equipe de cinco para três trabalhadores. As duas pessoas que sobraram foram inicialmente transferidas para outro setor, e demitidas cerca de um mês depois. Depois disso, o R8 se tornou evidente, pois os operadores resistiam a participar dos eventos kaizens, discutir os problemas do dia a dia ou sugerir melhorias. A empresa reconheceu o erro e levou alguns anos para conseguir novamente o apoio dos operadores.

Em 2006, com a troca da diretoria da empresa no Brasil, a relação entre o R11 (os operadores não se sentirem responsáveis pelo uso das práticas de PE) e o R9 (insegurança dos operadores na realização de novas atribuições) foi evidenciada. As melhorias 
foram realizadas pela equipe do setor de melhoria contínua e, em alguns momentos, ocorreram alterações em procedimentos e no arranjo físico, sem que os operadores participassem ou fossem avisados. A falta de envolvimento dos operadores na aplicação das práticas de PE, como na criação do trabalho padronizado e da troca rápida de ferramentas, fez com que eles não compreendessem o motivo do uso das práticas e muitas vezes não se sentissem confortáveis com as mudanças geradas. Esses dois riscos, R9 e R11, além de se relacionarem entre si, também causaram impacto no R8, pois a falta de conhecimento, treinamento e envolvimento dos operadores na aplicação das práticas de PE prejudicaram ainda mais o seu apoio.

De fato, os operadores não incorporavam a PE em sua rotina diária e algumas vezes interpretavam as práticas de $\mathrm{PE}$ como procedimentos burocráticos. Por exemplo, na visita à fábrica, percebeu-se que não havia uma preocupação no preenchimento dos quadros de acompanhamento de produção, pois estes estavam frequentemente incompletos e, dessa forma, os problemas não eram devidamente medidos e evidenciados.

\section{Conclusões}

A presente pesquisa teve como objetivo identificar e classificar os riscos na IPE. A identificação dos riscos foi realizada com base em uma revisão da literatura, a partir da qual 14 riscos foram definidos. Tal identificação exigiu esforço analítico, na medida em que foi necessário selecionar, dentre os estudos existentes, quais barreiras, dificuldades ou fatores de impacto no processo de implantação (termos usados na literatura) eram compatíveis com o conceito de risco adotado neste trabalho. Além disso, uma vez que é impossível identificar todos os riscos presentes em um processo, a lista de riscos identificada neste trabalho deve ser interpretada como uma base sujeita a refinamentos, especialmente em relação ao acréscimo de novos riscos e reorganização em diferentes níveis de abstração.

Já a classificação de riscos foi realizada com base em uma survey com 57 respondentes. Assim, os riscos identificados a partir da literatura foram classificados em três categorias, obtidas por meio de uma análise fatorial exploratória. Em que pese a limitação da amostra, os procedimentos usados na classificação dos riscos têm um caráter generalizável, e podem ser replicados em outros contextos. Além disso, a classificação dos riscos segundo os níveis hierárquicos associados a estes, embora tenha emergido da AFE, é coerente com o fato de a literatura apontar que as dificuldades de natureza gerencial e comportamental têm sido mais relevantes nos processos de IPE, em comparação às dificuldades de natureza técnica.
A natureza das relações entre os riscos que faziam parte de cada agrupamento identificado na AFE foi mais bem compreendida por meio de um estudo de caso em uma empresa na qual trabalhavam três respondentes da survey.

Vale salientar que a classificação de riscos pode contribuir para o direcionamento das ações de controle destes. É possível concluir que cada grupo representa um fator específico em função do forte inter-relacionamento entre os riscos dentro deles. Assim, uma mesma ação de controle e gerenciamento desses riscos tende a ter impacto sobre vários ou até todos os riscos de um mesmo grupo. Se no caso da empresa $A B C$, os operadores participassem da análise da situação atual e proposições futuras em eventos kaizen, por exemplo, todos os riscos relacionados ao G1 (Nível operacional) tenderiam a ser menores. A falta de envolvimento dos operadores foi identificada como um dos principais fatores que aumentaram os riscos de R8 (o nível operacional não apoiar a implantação), o R9 (insegurança dos operadores na realização de novas atribuições) e o R11 (os operadores não se sentirem responsáveis pelo uso das práticas de PE e pela solução de problemas). Uma outra ação, como a participação ativa da alta gerência através de seu envolvimento direto em acompanhar, priorizar as ações ou auxiliar no processo de implantação da PE, também tende a gerar uma redução nos riscos associados ao G2 (Alta e média gerência), uma vez que a reduziria diretamente o R6 (falta de apoio da média gerência) e R7 (falta de apoio da alta direção), e também garantiria uma alocação de recursos necessários para reduzir o R3 (falta de recursos destinados ao processo). No G1 (Áreas de apoio), um extensivo treinamento aos gestores de áreas ligadas à produção, logística, suprimentos, manutenção, engenharia e qualidade, por exemplo, pode reduzir vários riscos simultaneamente, como diretamente o R12 (os gerentes e diretores não terem o conhecimento suficiente sobre a PE) e R2 (falta de conhecimento das áreas de apoio para orientar o andamento da IPE), dando subsídios para que os indicadores de desempenho sejam modificados para estarem de acordo com os princípios lean, também reduzindo o risco R5 (dificuldades de enxergar o retorno financeiro nas ações realizadas). Também no $\mathrm{G} 1$, algumas iniciativas amplas e frequentes de marketing interno, com reportagens em jornais da empresa, por exemplo, visando divulgar a implantação da PE e os benefícios, podem reduzir o risco R4 (falta de clareza na comunicação para toda a empresa sobre o início e o progresso da implantação), melhorar a motivação dos colaboradores a participar das atividades de melhoria, reduzindo também R1 (desmotivação dos envolvidos após as primeiras mudanças), e ajudando a sustentar (R13) e manter o andamento (R14) do processo de implantação lean. 
As limitações e resultados desta pesquisa abrem espaço para futuros estudos envolvendo os temas PE e gestão de riscos, tais como: (a) a realização de surveys similares em outras regiões do país ou exterior, ou a realização de surveys periódicas junto a empresas de uma mesma região, para permitir a identificação de tendências setoriais e geográficas; (b) aprofundar o conhecimento sobre cada um dos riscos na IPE, assim como identificar as contramedidas que podem ser utilizadas; (c) a partir da definição clara do papel de cada nível hierárquico na gestão dos riscos, poderiam ser investigados os conhecimentos tácitos de cada um e, posteriormente, serem desenvolvidos métodos de capacitação e desenvolvimento ligados a tais conhecimentos; (d) o desenvolvimento de modelos descritivos acerca das relações entre os riscos, estratégias de controle destes e características do contexto organizacional que intensificam ou amenizam os riscos à IPE.

\section{Referências}

Achanga, P., Shehab, E., Roy, R., \& Nelder, G. (2006). Critical success factors for lean implementation within SMEs. Journal of Manufacturing Technology Management, 17(4), 460-471. http://dx.doi.org/10.1108/17410380610662889.

Aloini, D., Dulmin, R., \& Mininno, V. (2012). Risk assessment in ERP projects. Information Systems, 37(3), 183-199. http://dx.doi.org/10.1016/j.is.2011.10.001.

Baker, P. (2002). Why is lean so far off? Works Management, 55(10), 6-15.

Bannerman, P. L. (2008). Risk and risk management in software projects: a reassessment. Journal of Systems and Software, 81(12), 2118-2133. http://dx.doi.org/10.1016/j. jss.2008.03.059.

Bateman, N., \& David, A. (2002). Process improvement programmes: a model for assessing sustainability. International Journal of Operations \& Production Management, 22(5), 515-526. http://dx.doi.org/10.1108/01443570210425156.

Bayo-Moriones, A., Bello-Pintado, A., \& Merino-Díaz-DeCerio, J. (2008). The role of organizational context and infrastructure practices in JIT implementation. International Journal of Operations \& Production Management, 28(11), 1042-1066. http://dx.doi.org/10.1108/01443570810910188.

Bayou, M. E., \& Korvin, A. (2008). Measuring the leanness of manufacturing systems - a case study of ford motors company and general motors. Journal of Engineering and Technology Management, 25(4), 287-304. http://dx.doi. org/10.1016/j.jengtecman.2008.10.003.

Bhasin, S. (2012). Prominent obstacles to lean. International Journal of Productivity and Performance Management, 61(4), 403-425. http://dx.doi.org/10.1108/17410401211212661.

Black, J. (2007). Design rules for implementing the Toyota production system. International Journal of Production Research, 45(16), 3639-3664. http://dx.doi. org/10.1080/00207540701223469.

Blanchard, D. (2007). Lean, green and low cost. Industry WeekCleveland Ohio, 256(10), 37.

Cronbach, L. (1951). Coefficient alpha and the internal structure of tests. Psychiatrika, 16(3), 297-334.
Cua, K. O., McKone, K., \& Schroeder, R. G. (2001). Relationships between implementation of TQM, JIT, and TPM and manufacturing performance. Journal of Operations Management, 19(6), 675-694. http://dx.doi.org/10.1016/ S0272-6963(01)00066-3.

Emiliani, M., \& Stec, D. (2005). Leaders lost in transformation. Leadership and Organization Development Journal, 26(5), 370-387. http://dx.doi.org/10.1108/01437730510607862.

Farris, J., Van Aken, E., Doolen, T., \& Worley, J. (2009). Critical success factors for a human resource outcomes in Kaizen events: An empirical study. International Journal of Production Economics, 117(1), 42-65. http://dx.doi. org/10.1016/j.ijpe.2008.08.051.

Field, A. (2005). Discovering statistics using SPSS (2. ed.). London: Sage Publications.

Freitas, W. R. S., Jabbour, C. J. C., Teixeira, A. A., \& Jabbour, A. B. L. S. (2014). Gestão de recursos humanos e manufatura enxuta: evidências empíricas do setor automotivo brasileiro. Production, 24(2), 451-461. http://dx.doi.org/10.1590/ S0103-65132013005000039.

Golhar, D., Stamm, C., \& Smith, W. (1990). JIT implementation in small manufacturing firms. Production and Inventory Management Journal, 31(2), 44-48.

Hair, J. F., Black, B., Babin, B., Anderson, R., \& Tatham, R. (2006). Multivariate data analysis (6. ed ). New Jersey: Prentice Hall.

Hallikas, J., Karvonen, 1., Pulkkinen, U., Virolainen, V., \& Tuominen, M. (2004). Risk management processes in supplier networks. International Journal of Production Economics, 9O(1), 47-58. http://dx.doi.org/10.1016/j. ijpe.2004.02.007.

Hines, P., Holweg, M., \& Rich, N. (2004). Learning to evolve: A review of contemporary lean thinking. International Journal of Operations \& Production Management, 24(10), 994-1011. http://dx.doi.org/10.1108/01443570410558049.

Krafcit, J. F. (1988). Triumph of the lean production system. Sloan Management Review, 30(1), 41-51.

Lander, E., \& Liker, J. (2007). The Toyota production system and art: making highly customized and creative products at Toyota way. International Journal of Production Research, 45(16), 3681-3698. http://dx.doi.org/10.1080/00207540701223519.

Lean Enterprise Institute. (2007). New survey: middle managers are biggest obstacle to lean enterprise. Recuperado em 19 de outubro de 2007, de http://www.lean.org

Lian, Y., \& Van Landeghem, H. (2007). Analyzing the effects of Lean manufacturing using a value stream mappingbased simulation generator. International Journal of Production Research, 45(13), 3037-3058. http://dx.doi. org/10.1080/00207540600791590.

Lima, M. L., \& Zawislak, P. A. (2003). A produção enxuta como fator diferencial na capacidade de fornecimento de PMEs. Production, 13(2), 57-69. http://dx.doi.org/10.1590/ S0103-65132003000200006.

Malhotra, M. K., \& Grover, V. (1998). An assessment of survey research in POM: from constructs to theory. Journal of Operations Management, 16(4), 407-425. http://dx.doi. org/10.1016/S0272-6963(98)00021-7.

Malhotra, N. (2004). Marketing research: an applied orientation (4. ed.). New Jersey: Pearson/Prentice Hall.

Marodin, G. A., \& Saurin, T. A. (2013). Implementing lean production systems: research areas and opportunities for future studies. International Journal of Production Research, 51(22), 6663-6680. http://dx.doi.org/10.1080/ 00207543.2013 .826831 . 
Marodin, G. A., Saurin, T. A., \& Fettermann, D. C. (2014). Uma sistemática para a avaliação de riscos na implantação de produção enxuta. Revista Produção Online, 14(1), 364401. http://dx.doi.org/10.14488/1676-1901.v14.i1.1667.

Mathaisel, D. (2005). A lean architecture for transforming the aerospace maintenance, repair and overhaul (MRO) enterprise. International Journal of Productivity and Performance Management, 54(8), 623-644. http://dx.doi. org/10.1108/17410400510627499.

Meyers, L., Gamst, G., \& Guarino, A. (2006). Applied multivariate research. Thousand Oaks: Sage Publications.

Moreira, M., \& Alvez, R. (2008). A study on just-in-time implementation in Portugal: some empirical evidence. Brazilian Journal of Operations \& Production Management, 5(1), 5-22.

Motwani, J. (2003). A business process change framework for examining Lean manufacturing: a case study. Industrial Management \& Data Systems, 103(5), 339-346. http:// dx.doi.org/10.1108/02635570310477398.

Norrman, A., \& Jansson, U. (2004). Ericsson's proactive supply chain risk management approach after a serious sub-supplier accident. International Journal of Physical Distribution \& Logistics Management, 34(5), 434-456. http://dx.doi. org/10.1108/09600030410545463.

Nunnally, J. C. (1978). Psychometric theory (2. ed.). New York: McGraw-Hill.

Panizzolo, R. (1998). Appying lessons learned from 27 lean manufactures: the relevance of relationship management. International Journal of Production Economics, 55(3), 223240. http://dx.doi.org/10.1016/S0925-5273(98)00066-8.

Panizzolo, R., Garengo, P., Sharma, M. K., \& Gore, A. (2012). Lean manufacturing in developing countries: evidence from Indian SMEs. Production Planning and Control, 23(10-11), 769-788. http://dx.doi.org/10.1080/095372 87.2011.642155.

Papadopoulou, T., \& Özbayrak, M. (2005). Leanness experiences from the journey to date. Journal of Manufacturing Technology Management, 16(7), 784-807. http://dx.doi. org/10.1108/17410380510626196.

Pettersen, J. (2009). Defining lean production: some conceptual and practical issues. The TQM Journal, 21(2), 127-142. http://dx.doi.org/10.1108/17542730910938137.

Piercy, N., \& Rich, N. (2009). Lean transformation in the pure service environment the case of the call service center. International Journal of Operations \& Production Management, 29(1), 54-76. http://dx.doi.org/10.1108/01443570910925361.

Project Management Institute. (2004). A guide to the project management body of knowledge (3. ed.). Newtown Square: PMI.

Raz, T., Shenhar, A., \& Dvir, D. (2002). Risk management, Project success and technological uncertainity. $R \& D$ Management, 32(2), 101-109. http://dx.doi.org/10.1111/1467-9310.00243.

Ritchie, B., \& Brindley, C. (2000). Disintermediation, disintegration and risk in the SME global supply chain. Management Decision, 38(8), 575-583. http://dx.doi. org/10.1108/00251740010378309.

Ritchie, B., \& Brindley, C. (2007). Supply chain risk management and performance: a guiding framework for future development. International Journal of Operations \& Production Management, 27(3), 303-322. http://dx.doi. org/10.1108/01443570710725563.
Saurin, T. A., Ribeiro, J. L. D., \& Marodin, G. A. (2010). Identificação de oportunidades de pesquisa a partir de um levantamento da implantação da produção enxuta em empresas do Brasil e do exterior. Gestão \& Produção, 17(4), 829-841. http:// dx.doi.org/10.1590/S0104-530X2010000400015.

Scherrer-Rathje, M., Boyle, T. A., \& Deflorin, P. (2009). Lean, take two! Reflections from the second attempt at lean implementation. Business Horizons, 52(1), 79-88. http:// dx.doi.org/10.1016/j.bushor.2008.08.004.

Serviço Brasileiro de Apoio às Micro e Pequenas Empresas. (2011). Critérios e conceitos para classificação de empresas. Goiânia: SEBRAE. Recuperado em 3 de novembro de 2011, de http://www.sebrae.com.br/uf/goias/indicadores-das$\mathrm{mpe} /$ classificacao-empresarial

Shah, R., \& Ward, P. (2003). Lean manufacturing: context, practice bundles, and performance. Journal of Operations Management, 21(2), 129-149. http://dx.doi.org/10.1016/ S0272-6963(02)00108-0.

Sim, K., \& Rogers, J. (2008). Implementing lean production systems: barriers to change. Management Research News, 32(1), 37-49. http://dx.doi.org/10.1108/01409170910922014.

Sriparavastu, L., \& Gupta, T. (1997). An empirical study of JIT and TQM principles implementation in manufacturing firms in the USA. International Journal of Operations \& Production Management, 17(12), 1215-1232. http://dx.doi. org/10.1108/01443579710182954.

Sumner, M. (2000). Risk factors in enterprise-wide/ERP projects. Journal of Information Technology, 15(4), 317-327. http:// dx.doi.org/10.1080/02683960010009079.

Tabachnick, B. G., \& Fidell, L. S. (2001).Using multivariate statistics (4 ed.). Boston: Allyn \& Bacon.

Taj, S., \& Morosan, C. (2011). The impact of lean operations on the Chinese manufacturing performance. Journal of Manufacturing Technology Management, 22(2), 223-240. http://dx.doi.org/10.1108/17410381111102234.

Tang, C. (2006). Perspectives in supply chain risk management. International Journal of Production Economics, 103(2), 451-488. http://dx.doi.org/10.1016/j.ijpe.2005.12.006.

Turesky, E. F., \& Connell, P. (2010). Off the rails: understanding the derailment of a Lean manufacturing initiative. Organizational Management Journal, 7(2), 110-132. http:// dx.doi.org/10.1057/omj.2010.14.

Umbach, P. (2004). Web surveys: best practices. In J. F. Ryan \& G. Crisp (Eds.), New directions for institutional research (pp. 23-38). New York: Wiley On-line Library.

Wallace, L., Keil, M., \& Rai, A. (2004). Understanding software project risk: a cluster analysis. Information \& Management, 42(1), 115-125. http://dx.doi.org/10.1016/j.im.2003.12.007.

White, R. E., \& Prybutok, V. (2001). The relationship between JIT practices and type of production system. OMEGA: The International Journal of Management Science, 29(2), 113124. http://dx.doi.org/10.1016/S0305-0483(00)00033-5.

Williams, R., Bertsch, B., Dale, B., van der Wiele, T., van lwaarden, J., Smith, M., \& Visser, R. (2006). Quality and risk management: what are the key issues? The TQM Magazine, 18(1), 67-86. http://dx.doi.org/10.1108/09544780610637703.

Womack, J., \& Jones, D. (1996). Lean thinking: banish waste and create wealth in your corporation. New York: Simon and Schuster.

Yin, R. (2003). Case study research: design and methods (5. ed.). Thousand Oaks: Sage Publications. 


\section{ldentifying and classifying the risks involved in the process of implementing lean production}

\section{Abstract}

The process of implementing lean production (LP) is complex, slow, and subject to several risks. This article aims at identifying and classifying the risks involved in the LP implementation process. The study was undertaken in four stages: (i) a literature review to identify the risks; (ii) the design and application of a questionnaire, answered by 57 respondents, to assess their perceptions on the probability of occurrence and impact of the risks identified from the literature review; (iii) analysis of the results of the questionnaire, which allowed for classification of the risks based on an exploratory factor analysis; and (iv) a case study, to gain an understanding of the relationships among the risks, performed at a company for which three respondents of the survey worked. Fourteen risks were identified and then classified in three groups: (i) risks associated with the role of support areas; (ii) risks associated with the roles of the top management and middle management; and (iii) risks associated with the role of the operational level. Because the risks within each group are strongly interrelated, this classification can contribute to improving the lean implementation strategies by managing the risks.

\section{Keywords}

Lean Production. Risk management. Exploratory factor analysis. 